\title{
Utilidad de la Cintigrafía con tecnesio pertecnactato en el diagnóstico del Divertículo de Meckel
}

\author{
Drs, Sergio Ceresa*, Julio Espinoza, Ana María Cofré**. \\ Dinw Ciucotto**. Enrique Olea***. Patriwion Vers.****
}

\begin{abstract}

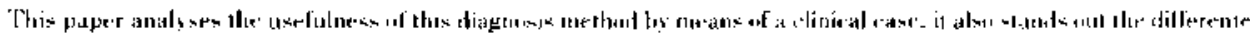

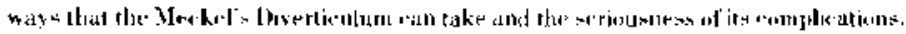

Se define el Divertíeulo de Meckel como la persistencia del conducto unfalomesentérico.

Se presenta en el 2 a $3 \%$ de lis individuos. Esrá ubicado en el ileon terminal, en el lado opuesto al mesenterio a poira distancia de la vályula jleocecal $(60$ a $90 \mathrm{~cm})^{1,2}$

El 25 a $30 \%$ de los individuos en que está presente, tienen sintomas rlínicrs. Estus se pueden manifestar en la infancia por sangramiento rectal acompanado a veces de otros síntomas idolor abdominal,vi-

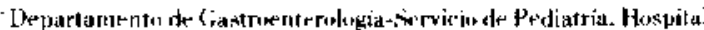
Patula Jaraduciumald,

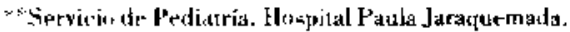

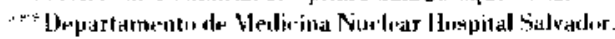

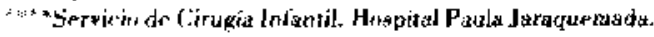

miltss, diarrea. fiebre, etc, t. El sangramientu digestivo resulta de la ulceración de la mucosa del diverlículo is en cl ileon adyacente, causado pur el jucido "horhídrico y pepsina secretados por mucesa gástriea ectípiea. La mucrsa gástrica er tópica está presente en lis mayoría de los diverticulos de pacientes con sangramiento intestinal. s. $^{3}$ to

Los esfuerzos para diagnesticar el Divertículo de Meckel mediante la radiongrafía y arteriografia soler. tiva han sido con fresuencia infructuosa."

Actualnjente se ha destacado la ulilidad de la rintigrafía ahdominal con 99 mTC pertec nactato en el diagnostico del Diverticulo de Meckel con mucosa gástrica ectípica: En aquellos en que está ésta ausente, el cirtigrama es falsamente negatiws. 


\section{CASO CLINICO}

R. R. H. lactante de un año y medio de edad, sexo masculino, presenta un desarrollo normal hasta log cuatros meses de edad en que se hospitaliza en Antofagasta por hemorragia digestiva (rectorragia). Al mes siguiente, es hospitalizado en otro servicio pediátrico de Santiago, por un episodio similar.

Cuatro días después del alta, presenta nuevamente rectorragia y deposiciones disgregadas por lo que se interna en nuestro Servicio el $7 /$ VI/78 con los diagnósticos de Obs. Enterocolitis Rectorragia-Anemia secundaria. Al examen fisico destacaba palidez de piel y mucosas ron Hto. 25\% por lo que requiere transfusión de $80 \mathrm{cc}$ de sangre. Evoluciona sin sangramiento aparente $y$ con normalización de depusiciones por to que se da de aha con control en Policlínica de Gastroenterología. Los exámenes realizados en ese momento (Tabla l) muestran a la Rectosigmoidoscopía mucosa rectal redundante y frágil. Evoluciona sin problemas hasta mayo/79 en que nuevamente presenta deposiciones con sangre roja, Hro control $31 \%$. Se practica nueva rectoscopía que demuestra fisura anal, esfínter anal hipotónico, mucosa rectal redundante y friable. Se mantiene sin sangramientos hasta 13 días más tarde, en que presenta melena, palidez de piel y mucosas, hospitalizândose nuevamente. Durante esa hospitalización presentó dos nuevos episodios de melena, llegando el Hto a $19 \%$ por lo que se transfunde 130 ce de glóbulos rojos. Las pruebas de coagulación y hepáticas on nomales (Tabla 2). El 7 de junio se practica examen con Tecnesio radiactivo, que es positivo (visualiza mucosa gástrica ectópica a nivel de îleon). (Fige. 1 y 2).

\section{Tabla 1}

Exámenes complementarios (junio 1978)

Hematocrito $\frac{7 / \mathrm{VT}}{25 \%}-\frac{8 / \mathrm{VI}}{31 \%} \frac{16 / \mathrm{VI}}{34 \%}$

Coprocultivo: E. coli tipificación (-)

Rx. Abdomen simple: normal

Tránsito intestinal: normal

Rectoscopia: Mucosa frágal que sangra al contacto con el instrumento. Redundante. Polipos (-).

Tabla 2

Exámenes complementarios (junio 1979)

Hematocrito $\frac{21 / \mathrm{V}}{21 \%} \mathrm{19 \%}$
Protrombina: $100 \%$
T. Cefalina: 38 seg.
Plaquetas: $128.000 \times \mathrm{mm}^{3}$
Pruebas Hepáticas: normales
Cintigrafía con Tecnesío: Visualiza mucosa gástri-
ca ectópica a nivel de ileon terminal.
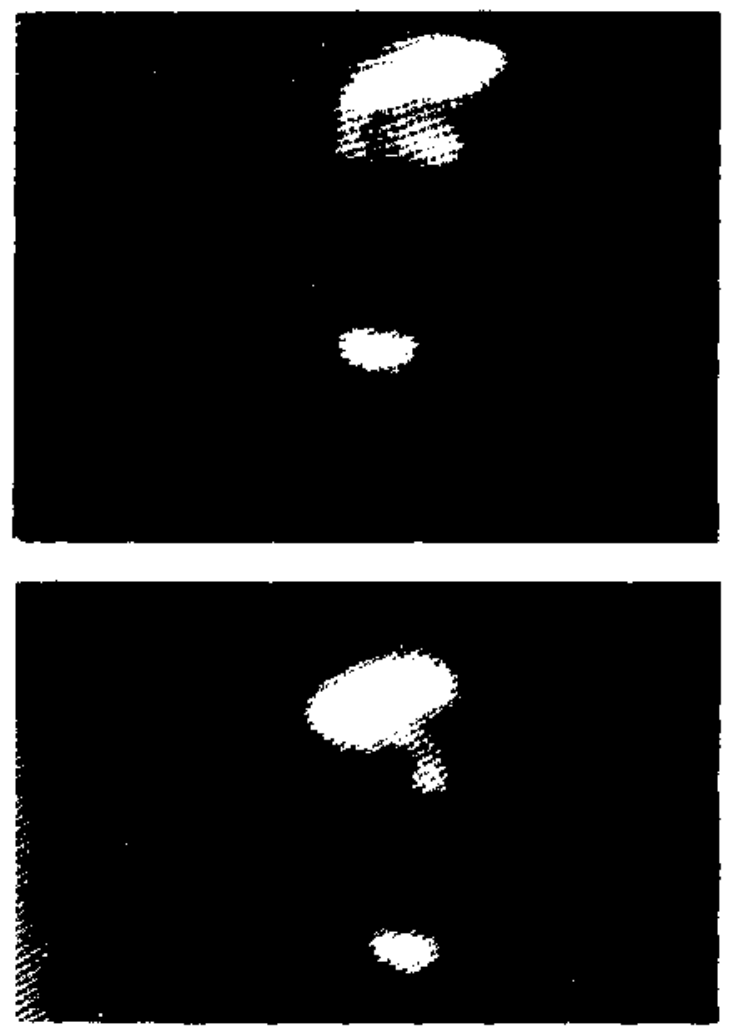

Figuras. 1 y 2. Cintierafia con Tecnesio en posición lateral y troatal que demuestra la presencia de Diverticulo de Mecke].

En la intervención quirúrgica, se encuentra " a $+30 \mathrm{~cm}$ de la válvula ileocecal, gran Divertículo de Meckel que compromete zona anterior y mesenterial, en extensión de ${ }^{+}$ $4 \mathrm{~cm}$. de forma cónica y en cuyo vértice se aprecia una coloración amarillenta. Se practica resección intestinal de $+7 \mathrm{~cm}$ que comprende todo el diverticulo dejando intestino sano para anastomosis término-terminal". La biopsja rápida informó segmento intestínal con Diverticulo de Meckel en el que se observa zona anular hemorrágica por estrangulaciôn. No se observan elementos neoplásicos ni otras alteraciones. (Figs. 3-).

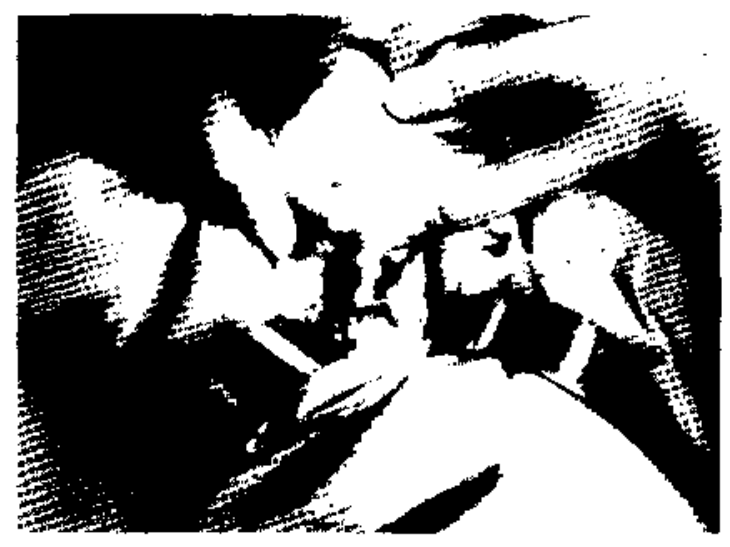

Figura 3. Preaencia de Diverticulo de Mecket en Deon Terminal. 
El exámen histológico posterior muestra zona de intlamación crónica con hiperhemia y cier to grado de fibrosis en la submucosa y con hemorragia en la serosa. Se visualiza mucusa gástriéa.

La evolución postoperatoria es satisfactoria, continuando asintomático en sus controles en Policlínica de Gastruenterología.

\section{COMENTARIO}

El Divertículo de Meckel es raro en la infancia, su diagnóstico es difícil y se manifiesta clínicamente por sus complicaciones que son la intlamación del divertículo simulando un proceso abdominal agudo, obstrucción intestinal, perforación y diverticulitis, hemorragia digestiva. En la mayoria de los r:asos el diagnóstico se realiza en el acto quirúrgico.

En un estudio retrospectivo realizedo en los Servicios de Cirugia, urgencia y anatomia patológica del Hospital Arriaránt se estudian los casos de Divertículos de Meckel encontrados entre 1943 y 1960، Los autores observaron un aumento de la incidencia de éste en el transcurso de los años alcanzando el $0.8 \%$. con amplio predominio del sexo masculino $(73.1 \%$ para diverticulo gano y $94.5 \%$ para divertículo patológico) ubicados entre $1.5 \mathrm{~cm}$ de la válvula ileocecal, de tamaños entre 0.4 y $8 \mathrm{~cm}$. El diverticulo sano se asociaba a otras malformaciones congénitas (42.6\%) siendo las más frecuentes las gastrointestinales, bazo supernumerario, compromiso del aparato urinario y cardiovascular. EI Divertículo de Meckel se manifestó en esa casuística, principalmente como obstrucción intestinal (66\%) e inflamación del divertículo con abdomen agudo. En un caso se observó melena que fue el único en que se sospechó tal diagnóstico preoperatorio. La conaulta tardía ( 2 a 7 días) determinó una elevada mortalidad $(71 \%)$.

Larraín y Danús comunican once casos de Divertículo de Meckel que consultaron por aus complicaciones, de los cuales cinco fallecen en el postoperatorio por la gravedad de ellas. En todos el Divertículo de Meckel fue un hallazgo quirúrgico.

En 1977 en una revisión hecha por Barraza y cols. ${ }^{5}$ de 40 niños operados de Diverticulo de Meckel, entre el año 1965 y 1976, se destaca la gravedad de sus complicaciones y la forma de presentación en el lac- tante: la obstrucción intestinal y la hemorragia digestiva. El $70 \%$ de los pacientes eran menores de 2 años.

Se ha descrito que el $50 \%$ de los Divertículos de Meckel tienen mucosa ectópica, siendo la mayoria mucosa gástrica ectópica. En los pacientes sintomáticos esta entidad se manifiesta por hemorragia digestiva caracterizada por sangramiento rectal.

El uso de la Cintigrafía con Tecnesio pertecnatato es de utilidad para el diagnóstico del Divertículo de Meckel con hemorragia digestiva, previo descarte de otras patologías más habituales como: pólipo rectal, fisura anal, enterocolitis, etc. El estudio radiológico con Bario y la endoscopía no deben ser realizados previo a la Cintigrafia, porque pueden causar hiperhemia de la mucosa intestinal, acumulación del 99 mmTC e imágenes de falsos positivos. 10

\section{RESUMEN}

Se analiza la utilidad de este método diagnóstico ilustrándose con un caso clinico, se destacan las distintas formas de presentación del Divertículo de Meckel y la gravedad de sus complicaciones.

\section{REFERENCLAS}

1 Nelson, W. E. Tratado de Pediatria. Salyat 1971.

2 Siluetmann, A., Roy, C. Ped. Cl, Gastromterokge St. Louis, Thi. C. U. Mosby Co., 1975.

3 Birquist, Th. Specificity of 99 mTC Pertechnetgle in scientigraphic diagnosis of Meckel's Diverticulum. Review of 100 cases. Journal of Nuclear Medicine. 17: 465-468. 1976.

4 Tanscher, Falge pusitive scan for Meckel's Diverticulum. The Journal of Pediatrics 92: 1022-1023, 1978.

5 Bartaza, P., Schalbe, K. "Corpplicaciones del Diverticulo de Meckel en la infancia". Séptimas Jomadas anuales de la Srciedad Chilena de Cirugja Infantil. Cancepción nov. 7i. (No publicado).

6 Muneyuki, Y. et al. Mectel'. Diverticulum. am. Journal of Sur. 136: $247-249,1978$.

7 Hansel, M. de Bartolo, Jonathan, A. Van Heerden, Meckel's Diverticulum. Am. Surc, Jan. 1976,

8 Lartaín, F. Dontús, $O$. Complicaciones del Diperticulo de Meckel. Análisis clínico higtopstológeón de 11 rosos. Rev. Ch. de Ped. 45: 451, 1974.

${ }^{9}$ Kilpatrick, T. M. Aseron, C. A. Radioisotope detection of Meckel's Diverticulum causing meute rexthl hemormge. N. End. J. Med. 287: 653, 1972.

10 Craft, A. W., Watson, A.J.Giant. Meckel's Diverticulum cauging intestinal obstruction in the newborn. J. of Ped. Surg. 1]: 1032. 1976.

11 Vera, M., Camacho, E. Espinoza, J. Divertículo de Meckel y ahdomen agudo quirúrzico. Rev. Chil. de ped. 32: 395, 1961. 